\title{
Demonstration of ALBidS: Adaptive Learning Strategic Bidding System
}

\author{
Tiago Pinto ${ }^{1}$, Zita Vale ${ }^{1}$, Isabel Praça ${ }^{1}$, Gabriel Santos ${ }^{1}$ \\ ${ }^{1}$ GECAD - Knowledge Engineering and Decision-Support Research Center, Institute of \\ Engineering - Politechnic of Porto (ISEP/IPP), Porto, Portugal \\ \{tmcfp, zav, icp, gajls\}@isep.ipp.pt
}

\section{Introduction}

Current worldwide electricity markets are strongly affected by the increasing use of renewable energy sources [1]. This increase has been stimulated by new energy policies that result from the growing concerns regarding the scarcity of fossil fuels and their impact in the environment. This has also led to an unavoidable restructuring of the power and energy sector, which was forced to adapt to the new paradigm [2]. The restructuring process resulted in a deep change in the operation of competitive electricity markets. The restructuring made the market more competitive, but also more complex, placing new challenges to the participants, which increases the difficulty of decision making. This is exacerbated by the increasing number of new market types that are being implemented to deal with the new challenges. Therefore, the intervenient entities are relentlessly forced to rethink their behaviour and market strategies in order to cope with such a constantly changing environment [2].

So that these entities can deal with the new challenges, the use of decision support tools becomes crucial. The need for understanding the market mechanisms and how players' interaction affects the outcomes of markets has contributed to the emergence of several simulation tools [3]. Multi-agent based software is the most widely adopted solution as this paradigm is particularly suitable to analyse dynamic systems with complex interactions among its elements, such as electricity markets. Current software tools allow studying different electricity market mechanisms and analysing the relationships between market entities; however, they are not prepared to provide suitable decision support to the negotiation process of electricity market players [4].

This gap motivates the development of this work, which arises with the purpose of providing a solution to enable electricity market players to take the best possible outcomes out of each market context. This contribution is provided by ALBidS (Adaptive Learning strategic Bidding System), a decision support system that includes a large number of distinct market strategies, and learns which should be used in each context in order to provide the best expected response [5]. ALBidS is also integrated with MASCEM (Multi-Agent Simulator of Competitive Electricity Markets), which enables the simulation of realistic scenarios using real data [6].

This project has received funding from the European Union's Horizon 2020 research and innovation programme under the Marie Sklodowska-Curie grant agreement No 641794. 


\section{Main Purpose}

The literature offers a large variety of strategic approaches that aim at providing decision support in auction-based electricity market negotiations [7]. However, none of the proposed strategies has shown to be clearly better than the others. Case studies show that there is no strategy that presents the best performance in all environments and contexts, i.e. strategies that have performed well in one situation may have mediocre performances in different circumstances [5].

The main goal of ALBidS is to take the most advantage out of the alternative market strategies that have been introduced in the literature. With this purpose, the general concept behind ALBidS is the integration of as many distinct market strategies as possible, whose performance is evaluated under different contexts of negotiation. This evaluation is used by the system to learn which strategies are the most adequate and present the highest chance of success in each different context.

The learning process is undertaken by means of reinforcement learning algorithms, namely the Roth-Erev algorithm and an algorithm based on the Bayesian theorem of probability [5]. Additionally, a 2E balance management mechanism has been developed to enable controlling the efficiency and effectiveness of the large variety of algorithms that are executed simultaneously. This method allows ALBidS to adapt the execution time of the system to the purpose of each simulation, e.g. if the expected results from ALBidS are as best as it is able to achieve, or if the main requirement is for the system to be executed rapidly to analyse issues other than player's optimal performance in the electricity market.

ALBidS incorporates a large variety of market decision support strategies with different natures and perspectives, such as data mining techniques, forecasting methods, artificial intelligence methodologies, application of electricity market directed strategies, mathematic approaches, economic theory based models, the adaptation of physics theories, game theory, metalearners, among others. This way, the system is able to take advantage of the characteristics of each approach whenever they show to be advantageous. The system is, thus, prepared to deal with different contexts and scenario situations, guaranteeing a large scope of approaches, which offer a greater chance of having appropriate responses even in very distinct situations.

The context awareness capabilities of ALBidS are provided by the context analysis methodology proposed in [8]. Different characteristics of each negotiation period and day are analysed so that different negotiation contexts are identified and defined. This methodology enables the learning process of ALBidS to be dependent on the negotiation context by adapting the responses to each current context. Strategies can, this way, be evaluated and chosen depending on their performance in each context.

The interaction with the MASCEM electricity market simulator provides the means for experimenting the developed decision support methods under realistic simulation conditions [6]. MASCEM makes use of the RealScen scenarios generator [9], to create simulation scenarios that are the representation of real markets. RealScen uses real data that is available online, which is gathered in real time, as soon as it is made available by each different source, using an automatic data extraction tool [9]. 


\section{Demonstration}

ALBidS is connected with the MASCEM simulator, providing a response to the negotiating players when they require intelligent support to act in the market. The connection between the two systems is managed by the Main Agent. This agent acts as an intermediary between the two systems. It receives requests from the negotiating players when they require decision support, and provides them the corresponding answers. These answers are provided after managing the ALBidS internal mechanism, including the interactions with the strategy agents. Fig 1 presents the user interface of ALBidS, which allows defining the inputs of the system and where the outputs of the decision support process can be consulted and analysed.

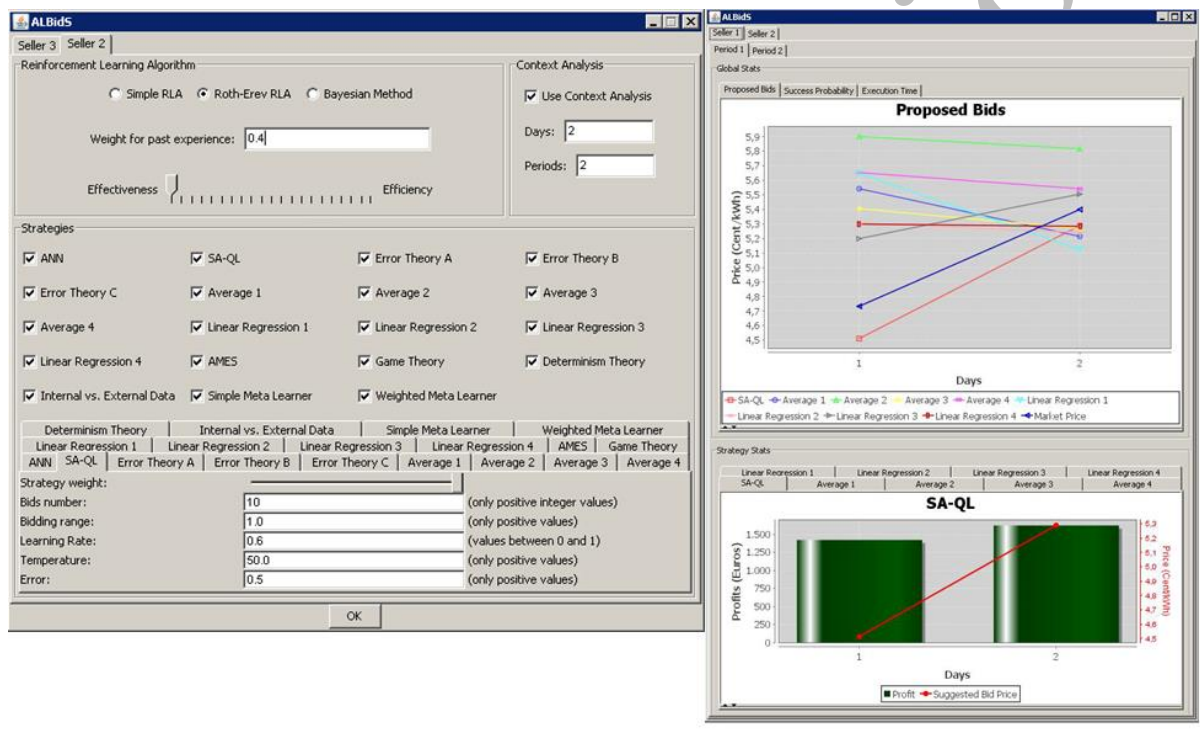

Fig. 1. ALBidS' user interface

From Fig. 1 it is visible that ALBidS enables choosing the desired reinforcement learning algorithm and defining its parameters. It is also possible to define the efficiency/effectiveness balance preferences and to set up the context analysis. In the strategy panel, it is possible to select the strategies to use, and define their parameters.

After all parameterizations are defined, and the simulation is started, the ALBidS interface displays the graphs presenting the simulation data. The interface displays two types of graphs: the global stats graphs, providing a global view of the simulation; and the strategy individual graphs, presenting the information regarding each of the strategies. In the global stats panel, three graphs are displayed, presenting: all strategies' bids and the actual market price, comparing the different strategies' proposals; the performance of each strategy; and the comparison of the execution times of all strategies. The second panel presents the individual strategy graphs, displaying for each one the bid prices and the obtained profits. 


\section{Conclusion}

The constant growth in electricity markets unpredictability resulted in an amplified need for market intervenient entities in foreseeing market behaviour. The need for understanding the market mechanisms and how the involved players' interaction affects the outcomes of the markets contributed to the growth of usage of simulation tools. Multi-agent based software is particularly well fitted to analyze dynamic and adaptive systems with complex interactions, such as electricity markets.

This paper presents a demonstration of ALBidS, a multiagent system created to provide decision support to market negotiating players. This system is integrated with the MASCEM electricity market simulator, so that its advantage in supporting market players can be tested using cases based on real markets' data.

ALBidS considers several different methodologies based on very dístinct approaches, to provide alternative suggestions of which are the best actions for the supported player to perform. The approach chosen as the players' actual action is selected by the employment of reinforcement learning algorithms, which for each different situation, simulation circumstances and context, decides which proposed action is the one with higher possibility of achieving the most success.

\section{References}

1. Sioshansi, F.P., "Evolution of Global Electricity Markets - New paradigms, new challenges, new approaches", Academic Press, 2013

2. Biggar, D.R. and Hesamzadeh, M.R. (Eds.), "The Economics of Electricity Markets", Wiley, 1st edition, September 22, 2014

3. Li, H., Sun, J., Tesfatsion, L., "Testing Institutional Arrangements via Agent-Based Modeling: A U.S. Electricity Market Application", Computational Methods in Economic Dynamics, Dynamic Modeling and Econometrics in Economics and Finance, Springer, H. Dawid and W. Semmler (Eds.), 13, 135-158, 2011

4. Pinto, T., Barreto, J., Praça, I., Sousa, T.M., Vale, Z., Solteiro Pires, E.J., "Six Thinking Hats: A novel Metalearner for Intelligent Decision Support in Electricity Markets", Decision Support Systems, Elsevier, 79, 1-11 November, 2015

5. Pinto, T., Vale, Z., Sousa, T.M., Praça, I., Santos, G., Morais, H., "Adaptive Learning in Agents Behaviour: A Framework for Electricity Markets Simulation", Integrated Computer-Aided Engineering, IOS Press, 21, 4, 399-415, September 2014

6. Praça, I., Ramos, C., Vale, Z., Cordeiro, M., "MASCEM: A Multi-Agent System that Simulates Competitive Electricity Markets". IEEE Intelligent Systems, Special Issue on Agents and Markets, 18, 6, 54-60, 2003

7. David, A.K. and Wen F., "Strategic bidding in competitive electricity markets: A literature survey", IEEE Proceedings Power Engineering Society Summer Meeting, 4, 2168-2173, 2000

8. Pinto, T., Vale, Z., Sousa, T.M., Praça, I., "Negotiation Context Analysis in Electricity Markets", Energy, Elsevier, 85, 78-93, June, 2015

9. Teixeira, B., Silva, F., Pinto, T., Praça, I., Santos, G., Vale, Z., "Data Mining Approach to support the Generation of Realistic Scenarios for Multi-Agent simulation of Electricity Markets", 2014 IEEE Symposium on Intelligent Agents (IA) at the IEEE SSCI 2014 (IEEE Symposium Series on Computational Intelligence), Orlando, Florida, USA, 09-12 December, 2014 